\title{
Assessing the Static and Dynamic Sensitivity of a Commercial Off-the-Shelf Multicore Processor for Noncritical Avionic Applications
}

\author{
Pablo F. Ramos $\mathbb{D}^{1},{ }^{1}$ Vanessa C. Vargas $\left(\mathbb{D},{ }^{1}\right.$ Nacer-Eddine Zergainoh, ${ }^{2}$ \\ and Raoul Velazco ${ }^{2}$ \\ ${ }^{1}$ DEEE, Universidad de las Fuerzas Armadas ESPE, Sangolquí 170501, Ecuador \\ ${ }^{2}$ Laboratoire TIMA, Université Grenoble-Alpes, Grenoble 38400, France \\ Correspondence should be addressed to Pablo F. Ramos; pframos@espe.edu.ec
}

Received 9 March 2018; Revised 30 May 2018; Accepted 13 June 2018; Published 8 July 2018

Academic Editor: David Quesada

Copyright (C) 2018 Pablo F. Ramos et al. This is an open access article distributed under the Creative Commons Attribution License, which permits unrestricted use, distribution, and reproduction in any medium, provided the original work is properly cited.

\begin{abstract}
The present work assesses the radiation sensitivity of an affordable and performant COTS multicore processor for noncritical avionic applications. The target device is the Epiphany E16G301 multicore manufactured in $65 \mathrm{~nm}$ CMOS which integrates 16 processor cores. This device was selected due to its high performance, low power consumption, and affordability, allowing general public accessing to parallel computing. Additionally, the E16G301 is the coprocessor for parallel processing of the Parallella platform, which was considered by NASA researchers for onboard health management of the DragonEye UAS. The evaluation of the device is done using quantitative theory by means of radiation experiments with $14 \mathrm{MeV}$ neutrons to emulate the effects of high-energy neutrons present at avionic altitudes. Static and dynamic cross sections are obtained to evaluate the intrinsic sensitivity of the device as well as its dynamic response. Results show that the failure rate of the E16G301 running a matrix multiplication as application reaches level D of the DO-178B/C guideline, being the device well suited for minor failure conditions of avionic applications.
\end{abstract}

\section{Introduction}

Multicore processors are a suitable solution for achieving high performance and reliability without increasing significantly the power consumption. Its processing capacity and redundancy capabilities make them appropriate devices for implementing fault-tolerant mechanisms $[1,2]$. Hence, avionic industries are interested in incorporating these devices in their systems [3]. However, the high degree of miniaturization (nanometer-scale) of multicores increases their vulnerability to the effects of natural radiation. This radiation may result in transient and permanent failures called single event effects (SEEs). Among them, the single event upset (SEU) is the most representative, since it may produce the modification of the content of a memory cell [4]. For this reason, manufacturers are enhancing fabrication processes and architectural designs. Silicon-on-Insulator (SOI) is a clear example of technology improvements, implemented to face traditional bulk CMOS drawbacks $[5,6]$. Radiation hardening by design (RHBD) techniques are also used to mitigate SEU consequences [7]. The implementation of error correcting codes (ECCs) and parity to protect the internal memory of the processors is useful but not enough in presence of multiple bit upsets (MBUs). Another wellknown RHBD technique is the triple modular redundancy (TMR) which significantly improves the reliability of the system. Nevertheless, having more robust or dedicated components implies a considerable increase in costs. Consequently, an important challenge for aircraft industries is the integration of commercial-off-the-shelf (COTS) multicore processors due to budget and availability issues [8]. The current work assesses the effects of neutron radiation on a multicore processor which does not implement protection mechanisms in its internal memories. This is achieved by means of two accelerated 
radiation experiments. The first one aims at evaluating the device (hardware) sensitivity, while the second one evaluates the application (software) sensitivity. Part of the results of current research has been presented in [9].

\section{Related Work}

There are some works in the literature regarding accelerated radiation experiments on multi-/manycore processors. The most representative ones are given below.

Stolt and Norman established a dynamic cross section model for a multicore server based on quadcore processors built in $45 \mathrm{~nm}$ bulk CMOS technology. The target multicore was an HP c7000 BladeSystem designed for aircraft altitudes. Radiation experiments on the multicore server were conducted with the $14 \mathrm{MeV}$ neutron in order to simulate the effects of high energy particles present at avionic altitude. The server is composed by six Intel X5570-based HP server blades and six interconnect modules. For the test, it is possible to select the operating system, the BIOS setting, the processor, and the input/output utilization. Results estimate that the cross section per bit for $45 \mathrm{~nm}$ CMOS technology at $14 \mathrm{MeV}$ neutrons is $1 \times 10^{-14} \mathrm{~cm}^{2} /$ bit [10].

Guertin presents radiation experiments on the 49-core Maestro processor, which is a radiation hardened by design (RHBD) device for space applications based on the Tilera TILE64 processor. This $90 \mathrm{~nm}$ manycore is produced by the onboard processing expandable reconfigurable architecture (OPERA) program and built by the Boeing Solid State Electronics Development (SSED). Experimental tests have been conducted at the Texas A/M University's (TAMU) cyclotron facility using 15 and $25 \mathrm{MeV}$ ions. During the tests, internal registers as well as the $\mathrm{L} 1$ and $\mathrm{L} 2$ cache memories of the tile core were targeted. The main observed events were upsets in the L1 and L2 caches which were handled by an effective error correction and detection (EDAC) included in the Maestro design [11].

Santini et al. proposed a generic metric (mean workload between failures) to evaluate the reliability of an embedded processor intended to execute safety-critical applications. This study considers both cross section and exposure time for demonstrating that, on modern embedded processors, enabling the cache memories may provide benefits to critical systems in terms of reliability. This is possible since the larger exposed sensitive area may be compensated by a shorter exposure time of the application. The proposed metric is validated through extensive radiation test campaigns targeting a $28 \mathrm{~nm}$ COTS ARM-based SoC. The experiments were performed at the Los Alamos National Laboratory (LANL) and Los Alamos Neutron Science Center (LANSCE) with white neutrons source that emulates the energy spectrum of the atmospheric neutron flux. The failure probability of a bare-metal application is decreased when L1 cache is enabled. Consequently, it is not enough to rely only upon the cross section to ponder reliability [12].

Oliveira et al. presented the radiation sensitivity evaluation of cache memories and internal resources of modern graphic processing units (GPUs) designed in a $28 \mathrm{~nm}$ technology node. In addition, several hardening strategies based on duplication with comparison (DWC) to reduce GPU radiation sensitivity are presented and validated through radiation experiments. The device under test was NVIDIA K20 that contains a compute unified device architecture (CUDA-) -based GPU. The cross section per bit of the L2 cache and shared memories were experimentally obtained at the Los Alamos facility using $14 \mathrm{MeV}$ energy neutrons. Three different DWC strategies were designed to mitigate radiation-induced effects on GPU's used in safetycritical and high-performance computing (HPC) applications. The efficiency of the proposed strategies was experimentally evaluated and compared with chip's ECC protection mechanism. It was demonstrated that DWC strategies can be more effective than ECC when input data are duplicated [13].

Ramos et al. illustrated the radiation experiments on a quadcore processor built in a $45 \mathrm{~nm}$ SOI. The target device was the Freescale QorIQ P2041 processor, which is a highperformance device designed for communications. Experimental tests were conducted in the GENEPI2 (GEnerator of NEutron Pulsed and Intense) particle accelerator located in Grenoble, France. The results show that the SOI technology is between three and five times less sensitive to SEE than its CMOS counterpart. The dynamic asymmetric multiprocessing (AMP) tests have demonstrated that in spite of parity and ECC protection mechanisms, errors have been occurred in the result of the application. In addition, it can be seen that the dynamic sensitivity of the device strongly depends on the implemented multiprocessing mode [14].

Vargas et al. evaluated the SEE static and dynamic sensitivity of a manycore processor built in $28 \mathrm{~nm}$ CMOS. The target device was the Kalray MPPA-256 processor which is a power efficient device implementing a clustered architecture with 16 compute clusters each one with 16 processing elements. Radiation experiments were conducted in a GENEPI2 particle accelerator located in Grenoble, France. The evaluation of the device's dynamic response shows that, by enabling the cache memories, it is possible to gain in performance of the application without compromising reliability. Additionally, the results suggest that ECC and interleaving implemented in the static memories of the targeted clusters are very effective to mitigate SEUs since all detected events were corrected [15].

\section{Methodology and Materials}

Accelerated radiation ground testing allows performing the analysis of the sensitivity to radiation of electronic devices through artificial radiation environments. It is the fastest way to obtain statistically meaningful data in a short period of time, since the more particles hit the component, the more SEEs are observed [16]. The reproducibility of the experiment is also another major advantage of this strategy. Consequently, this work considers two models of tests for evaluating the sensitivity of a multicore processor: a static test in order to obtain the intrinsic sensitivity of the device's memory cells and a dynamic test for evaluating the dynamic response of the implemented application [17]. Figure 1 illustrates the proposed methodology. 


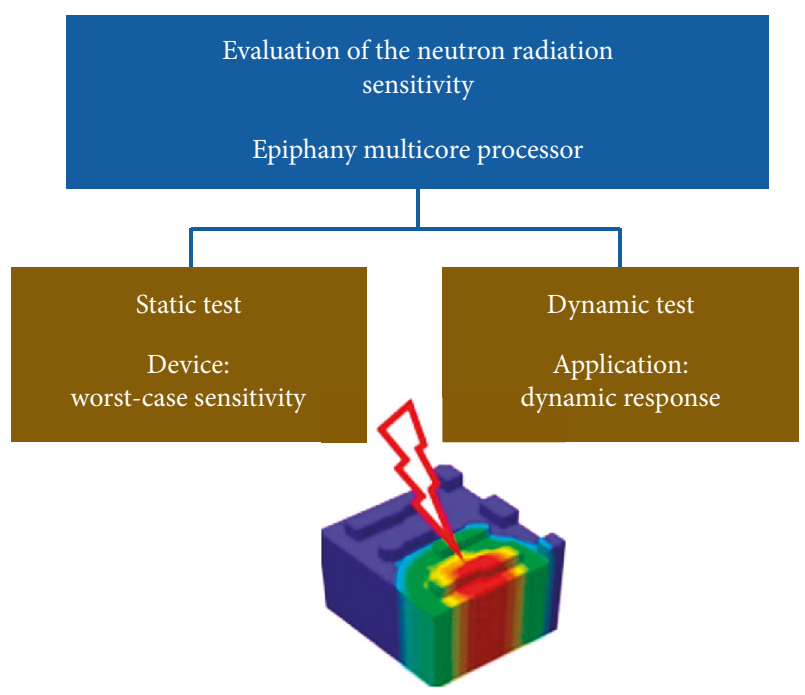

FIGURE 1: Multicore processor neutron sensitivity evaluation.

In this work, experimental tests have been conducted with $14 \mathrm{MeV}$ neutron radiation to emulate the effects of high-energy neutrons present at avionic altitudes, since neutrons are the most representative particles in the Earth's atmosphere. Reference [18] discusses the relevance of using the $14 \mathrm{MeV}$ neutron test to characterize the SEU sensitivity of digital devices. Sections 3 and 6 of the JESD89A document of the JEDEC standard were used as a base protocol for the experimental tests [19].

3.1. Identification of the Variables. Radiation tests are experiments that can be addressed using quantitative theory. Consequently, the first task is to identify the variables involved in the experiment. Table 1 lists the independent and dependent variables for the static and dynamic tests. Note that the dynamic test also depends on the system configuration and the implemented application.

The independent variables are divided in two groups: variables depending on the system (exclusive for the dynamic test) and variables to be manipulated during the radiation experiments.

Dependent variables represent the errors observed during the tests. They can be classified into single errors, multiple errors, and sequence interruption errors. It is important to consider that, depending on the memory architecture of the multicore, single and double errors can be corrected and detected, respectively, by the protection mechanisms.

3.2. Static Test. This test aims at estimating the intrinsic sensitivity to SEE of the memory cells of a processor. The device under test (DUT) is placed facing the center of the target perpendicularly to the beam axis at a distance depending on the required radiation flux. Typically, the method consists in writing a predefined pattern in the memory and accessible registers of the processor via the instruction set (load and store). Once finished the initialization, the DUT is irradiated and the program checks
TABLE 1: Independent and dependent variables for radiation tests.

\begin{tabular}{ccc}
\hline \multicolumn{2}{c}{ Independent variables } & Dependent variables \\
\hline & Neutron flux & \\
Manipulated (static & Distance DUT & Single-bit upset (SBU) \\
and dynamic tests) & to target & Multiple-bit upset \\
& $\begin{array}{c}\text { Exposure time } \\
\text { Implemented }\end{array}$ & (MBU) \\
& application & Multiple-cell upset \\
& (MCU) \\
$\begin{array}{c}\text { System dependent } \\
\text { (dynamic test) }\end{array}$ & $\begin{array}{c}\text { Multiprocessing } \\
\text { mode }\end{array}$ & $\begin{array}{c}\text { Single-event functional } \\
\text { interruption (SEFI) }\end{array}$ \\
& Programming & \\
& model & \\
\hline
\end{tabular}

periodically the memory locations along the radiation test to detect upset events. If an upset is detected, the program writes the correct pattern in the associated memory location and logs the results to an external host via Ethernet ports. During the static test, all the sensitive zones are exposed to radiation at the same time, which do not represent the real behavior of the circuit since not all the memory resources are used simultaneously when an application is executed. For this reason, the static test provides the worst-case estimation of the device sensitivity [20].

As a result of this test, the static cross section $\left(\sigma_{\text {STATIC }}\right)$ of the device is obtained. It is defined as the number of detected upset events divided by the fluence, which is the neutron flux integrated in time, as expressed in the following equation:

$$
\sigma=\frac{\text { number of SEE }}{\text { fluence }} \text {. }
$$

The elementary data pattern for memory circuits is a logical checkerboard [19]. All zeros and all ones is also a common pattern used during the radiation test. However, some memories such as DRAMs usually have a favorite error failure, either $0->1$ or $1->0$. For this reason, for testing when there is no a priori information about the component, the test pattern have to balance the number of 0's and 1's. Thus, the selected pattern for the static test was 0x55AA55AA. Regarding the exposure time to radiation of the device, it is important to consider that the probability of having an upset event during a given period of time is a stochastic process that follows a Poisson distribution. Thus, the waiting time between the read operations in the static test can be validated by analyzing the distribution of the number of events per unit of time. If the obtained distribution does not follow the Poisson law, the waiting time should be adjusted.

3.3. Dynamic Test. The goal of this test is to estimate the SEE dynamic response of an application running on a processor. As a result of the experiment, the dynamic cross section $\left(\sigma_{\text {DYN }}\right)$ is obtained. Unlike the static test, it only evaluates the memory cells used by the application. The method consists in the periodical execution of an application while the processor is being irradiated to induce SEE. Once finished each execution of the program, results are compared with a set of correct values previously obtained, in order to detect errors. The experiment is launched and monitored using a host computer located outside the armored chamber. The 


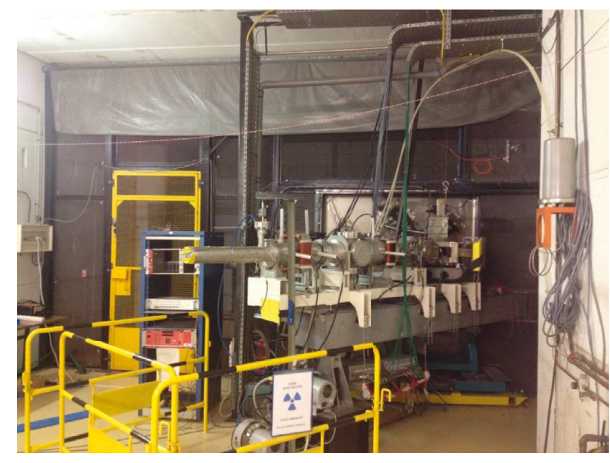

FIgURE 2: GENEPI2 particle accelerator.

communication between the external host and the Parallella host is achieved by means of the Ethernet port using the Linux ssh communication command. All detected errors are logged and transmitted to the external host which stores the results.

3.4. Neutron Radiation Facility. Accelerated radiation ground tests were conducted at the GENEPI2 (GEnerator of NEutron Pulsed and Intense) facility located at the LPSC (Laboratoire de Physique Subatomique et Cosmologie) in Grenoble, France. This accelerator was originally developed for nuclear physics experiments, and since 2014, it has been used to irradiate integrated circuits from different technologies. GENEPI2 is an electrostatic accelerator producing neutrons by impinging a deuteron beam onto a tritium (T) target. After acceleration at $220 \mathrm{keV}$, deuterons (d) produce neutrons ( $\mathrm{n}$ ) by the fusion reaction $\mathrm{d}+\mathrm{T} \rightarrow n+4 \mathrm{He}$ [21].

Neutrons are emitted omnidirectionally to the DUT with an average energy of $14 \mathrm{MeV}$. The DUT is set facing directly the target at a distance depending on the required neutron flux. Experimental radiation campaigns consider that only neutrons emitted fully forward will impact the DUT. For protecting the readout electronic platform other than the DUT, a dedicated neutron shielding is used.

A new $\mathrm{T}$ target providing a maximum neutron flux of $4.5 \times 10^{7} \mathrm{n} \cdot \mathrm{cm}^{-2} \cdot \mathrm{s}^{-1}$ was installed in 2015 aiming at increasing the neutron production while improving the accelerator reliability. The major modification consists in replacing the current deuterium ion source by a new one, based on the electron cyclotron resonance (ECR) technique, delivering higher-beam intensity. Figure 2 illustrates GENEPI2 particle accelerator.

3.5. Device under Test. The selected device for this study was the Adapteva Epiphany E16G301 which is a 16-core processor designed for parallel computing of the Parallella board. This board is a high-performance computing platform based on a dual core ARM A9 processor, used as host, and the Epiphany E16G301 used as coprocessor. The Epiphany is a scalable multicore architecture with up to 4095 processors sharing a common 32-bits memory space. It defines a parallel computing fabric comprised of a $2 \mathrm{D}$ array of processors nodes connected by a low-latency mesh network-on-chip. The E16G301, which is based on 3rd generation of the Epiphany multicore IP, is a 16-core system-on-chip implemented in a $65 \mathrm{~nm}$ CMOS technology [22]. Each processor core is a 32-bit superscalar floating point RISC CPUs, capable of performing two floating point operations per clock cycle and one integer calculation per clock cycle. The device has a peak performance of 32 Gflops (2 Gflops per core). The maximum chip power consumption is less than $2 \mathrm{~W}$. Each CPU has an efficient general-purpose instruction set that excels at compute-intensive applications while being efficiently programmable in $\mathrm{C} / \mathrm{C}++$. Figure 3 shows the implementation of the E16G301 architecture.

The memory architecture of the E16G301 multicore is based on a flat shared memory map. Each compute core has up to $1 \mathrm{MB}$ of local memory as a unique addressable part of the total 32-bit address space. The core processor can access its own local memory as well as other processors' memory by means of standard load/store instructions. The local memory is comprised of 4 independent banks, each one of $8 \mathrm{~KB}$ for a total of $32 \mathrm{~KB}$ for each CPU core. For the particular case of the Epiphany E16G301 that implements 16-cores, the chip has a $512 \mathrm{~KB}$ distributed shared memory [23]. This multicore processor does not implement any protection mechanisms in its internal memory.

3.6. Benchmark Application. A standard $45 \times 45$ matrix multiplication (MM), which is a memory-bound application, was selected to be tested throughout this work. It was considered since the matrix multiplication is one of the most essential algorithms in numerical algebra as well as in distributed, scientific, and high-performance computing [24]. Concerning avionic applications, $\mathrm{MM}$ is used for image processing, filtering, adaptive control, and navigation and tracking. The input matrix A was filled up with the decimal number " 5 ," while matrix B was filled up with $6 \mathrm{~s}$; thus the expected result was 1350 for all the elements of the resulting matrix C. The total number of variables used for the implementation of the matrix multiplication is 6078, distributed in 4050 input variables, 2025 output variables, and 3 indexes for loop operations. Each variable was implemented in 32-bit, being the targeted sensitive area about $24 \mathrm{~KB}$ that perfectly fits in the $32 \mathrm{~KB}$ local memory of each core. The size of the matrix was selected so that data occupy as much memory space as possible, leaving enough space for the program's code.

\section{Results and Discussion}

Radiation experiments performed on the Epiphany E16G301 are very interesting compared to similar works targeting other multicore processors, since errors produced by SEE are clearly identified as they are not masked by protection mechanisms such as ECC or parity. This fact allows a better analysis of the behavior of the device in presence of SEEs.

Concerning the limitations of the experiments, there are two points to consider:

(i) The E16G301 processor does not have direct access to printf function for logging results. For this reason, 


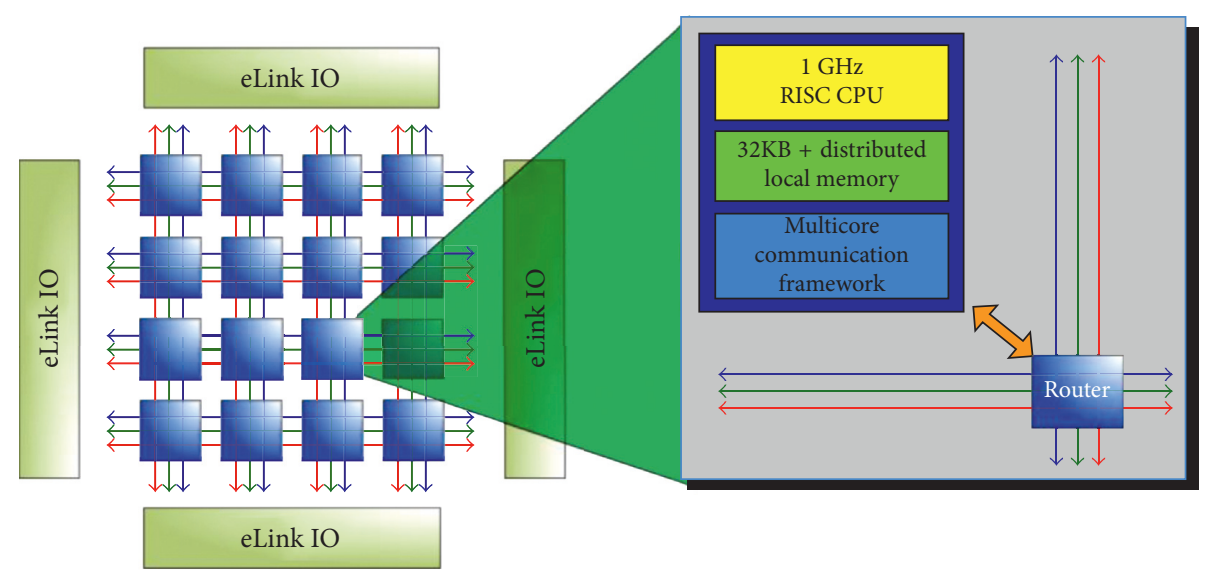

FIGURE 3: Implementation of the E16G301 Epiphany architecture [22].

it has to write the information about observed events in the external DDR memory of the board. This information is logged by the host processor (ARM).

(ii) The physical distance between the E16G301 multicore and the host processor in the Parallella board is less than one centimeter. It was thus necessary to limit the neutron flux for avoiding particles affecting the host processor and other circuitries.

4.1. Experimental Setup. The DUT was placed at a distance of $38.5 \pm 0.5 \mathrm{~cm}$ to the target. The neutron beam energy was $14 \mathrm{MeV}$ with an estimated flux of $7.2 \times 10^{4} \mathrm{n} \cdot \mathrm{cm}^{-2} \cdot \mathrm{s}^{-1}$ with an error of $\pm 0.1 \times 10^{4} \mathrm{n} \cdot \mathrm{cm}^{-2} \cdot \mathrm{s}^{-1}$. Special attention was required to protect the rest of the platform components from radiation. For that, the E16G301 multicore was irradiated through a small window on a $5 \mathrm{~cm}$ thickness polypropylene block intended to protect the readout platform. The power supplied of the multicore platform was monitored by using a camera available in the casemate of the accelerator facility. In this way, the voltage and current parameters were controlled.

4.2. Intrinsic Sensitivity. This evaluation was performed by targeting the internal memory and accessible registers of each core of the E16G301 processor. The host processor of the Parallella board was in charge of filling the internal memory and registers of the multicore with a predefined pattern using the Epiphany SDK utilities E-READ and E-WRITE [25]. In this manner, the whole internal memory of the E16G301 multicore could be targeted. Table 2 summarizes the sensitive zones of the multicore processor.

Three static tests were performed with an exposure time of 1 hour each one, providing a fluence of $6.82 \times 10^{8} \mathrm{n} \cdot \mathrm{cm}^{-2}$ as per the neutron facility records. During the tests, $69 \mathrm{SBU}$ and $7 \mathrm{MCU}$ that produce bit-flips were detected. There were no observed errors in processor's registers. In addition, 5 SEFI that caused hangs were observed. Table 3 summarizes the test results. Note that the subscript number following MCU represents the multiplicity of the upset.

Table 4 shows a sample of data containing bit-flips caused by SBU and MCU produced in the local memory and logged during the experiment.
TABle 2: Sensitive zones of the Epiphany E16G301 multicore processor.

\begin{tabular}{lccc}
\hline $\begin{array}{l}\text { Sensitive } \\
\text { zone }\end{array}$ & Location & Capacity & Description \\
\hline SRAM & $\begin{array}{c}\text { Processor } \\
\text { core }\end{array}$ & 32 KB per core & $\begin{array}{c}\text { Local memory } \\
\text { General- }\end{array}$ \\
GPR & $\begin{array}{c}\text { Processor } \\
\text { core }\end{array}$ & $\begin{array}{c}\text { 64 registers of 32 bits } \\
\text { per core }\end{array}$ & $\begin{array}{c}\text { purpose } \\
\text { register }\end{array}$ \\
SPR & $\begin{array}{c}\text { Processor } \\
\text { core }\end{array}$ & $\begin{array}{c}42 \text { registers of 32 bits } \\
\text { per core }\end{array}$ & $\begin{array}{c}\text { Special-purpose } \\
\text { register }\end{array}$ \\
\hline
\end{tabular}

TABLE 3: Results of the static radiation test campaigns.

\begin{tabular}{lcccc}
\hline SEE Type & Test 1 & Test 2 & Test 3 & Consequences \\
\hline SBU & 29 & 17 & 23 & Bit-flip \\
MCU (2) & 1 & 5 & 1 & Bit-flip \\
SEFI & 3 & 2 & 0 & Hangs \\
Total & 33 & 24 & 24 & - \\
\hline
\end{tabular}

At the end of the experiment, the static cross section $\sigma_{\text {STATIC }}$ was estimated by applying (1). It provides the worstcase sensitivity of the device built in $65 \mathrm{~nm}$ CMOS technology.

$$
\sigma_{\text {STATIC }}=\frac{81}{6.82 \times 10^{8}}=1.29 \times 10^{-7} \mathrm{~cm}^{2} / \mathrm{dev} .
$$

Due to the scarcity of experimental data, $95 \%$ confidence intervals were applied to this result. In this case, the most accurate way to calculate the uncertainty margins consists in using the relationship between the cumulative distribution functions of the Poisson and chi-squared distributions as described in [26]. Then, the lower and upper limits for the dynamic cross section are

$$
0.94 \times 10^{-7} \mathrm{~cm}^{2} / \mathrm{dev}<\sigma_{\text {STATIC }}<1.48 \times 10^{-7} \mathrm{~cm}^{2} / \mathrm{dev} .
$$

Since the tested memory area of the multicore processor represents 4194304 bits, the $95 \%$ confidence interval for the static cross section per bit is estimated as 
TABLE 4: Example of the obtained results in the static tests.

\begin{tabular}{lccccc}
\hline Pattern & Type & Core & Address & Data hex & Data binary \\
\hline & SBU & 3 & 0x7C84 & 0x55AA45AA & 0b01010101 101010100100010110101010 \\
0x55AA55AA & SBU & 7 & 0x52B4 & 0x55AA55AB & 0b01010101 1010 1010 0101 0101 1010 1011 \\
& MCU (2) & 2 & 0x7110 & 0x55AA5DAA & 0b01010101 1011 1010 0101 1101 1010 1010 \\
& & & 0x7130 & 0x55AA5DAA & 0b01010101 101110100101110110101010 \\
\hline
\end{tabular}

TABLE 5: Results of the dynamic radiation test campaigns.

\begin{tabular}{lcccc}
\hline SEE Type & Test 1 & Test 2 & Test 3 & Consequences \\
\hline SEU & 10 & 6 & 7 & Silent faults \\
SEU & 6 & 4 & 11 & Erroneous results \\
SEU & 1 & 0 & 0 & Time-outs \\
SEFI & 2 & 2 & 1 & Hangs \\
Total & 19 & 12 & 19 & - \\
\hline
\end{tabular}

$$
\sigma_{\text {STATIC_bit }}=[2.24-3.52] \times 10^{-14} \mathrm{~cm}^{2} / \mathrm{bit} \text {. }
$$

4.3. Dynamic Response. This evaluation was carried out to obtain the dynamic cross section $\left(\sigma_{\mathrm{DYN}}\right)$ of an application running in the multicore processor. Three dynamic tests were performed with an exposure time of 1 hour each one providing a total fluence of $6.95 \times 10^{8} \mathrm{n} \cdot \mathrm{cm}^{-2}$ as per the accelerator records. The comparison between the obtained and correct results was performed inside the multicore processor. The duration of the matrix multiplication is $27597 \mu \mathrm{s}$, and the comparison time is $1.44 \mu \mathrm{s}$. Therefore, in one hour (3600s), the application executes 130449 times, which multiplied by the comparison time gives $0.19 \mathrm{~s}$ $(0.0052 \%)$ of loss of exposure time which is negligible. Table 5 summarizes the results of the dynamic radiation campaign.

From the results presented in Table 5, only erroneous results, time-outs, and hangs were taken into account to calculate the dynamic cross section as follows:

$$
\sigma_{\text {DYNAMIC }}=\frac{27}{6.95 \times 10^{8}}=3.88 \times 10^{-8} \mathrm{~cm}^{2} / \mathrm{dev} \text {. }
$$

Silent errors were observed by reading input matrices aimed at detecting corrupted data caused by upset events which do not produce errors in the resulting matrix. They are presented for showing the total number of SEUs occurred in the dynamic test. As in the static case, uncertainty margins were added to the results. Then, the lower and upper limits for the dynamic cross section for a 95\% confidence interval are as follows:

$$
2.56 \times 10^{-8} \mathrm{~cm}^{2} / \mathrm{dev}<\sigma_{\mathrm{DYN}}<5.65 \times 10^{-8} \mathrm{~cm}^{2} / \mathrm{dev} .
$$

From the consequences of the dynamic tests, erroneous results are the most critical since the program considers them as valid results affecting dramatically the reliability of the application. The reliability of the device can be evaluated by means of its failure rate. The failure rate $(\lambda)$ of the device is estimated by extrapolating the dynamic cross section at avionic altitude $(35,000$ feet) where the neutron flux $(\varphi)$ is about $2.99 \times 10^{3} \mathrm{n} \cdot \mathrm{cm}^{-2} \cdot \mathrm{h}^{-1}$, by applying the following equation:
TABLE 6: Failure condition levels according to DO-178B/C $[28,29]$.

\begin{tabular}{lcccc}
\hline Level & $\begin{array}{c}\text { Failure } \\
\text { condition }\end{array}$ & Objectives & $\begin{array}{c}\text { With } \\
\text { independence }\end{array}$ & $\begin{array}{c}\text { Failure } \\
\text { rate }\end{array}$ \\
\hline A & Catastrophic & 66 & 25 & $P \leq 10^{-9} / \mathrm{h}$ \\
B & Hazardous & 65 & 14 & $P \leq 10^{-7} / \mathrm{h}$ \\
C & Major & 57 & 2 & $P \leq 10^{-5} / \mathrm{h}$ \\
D & Minor & 28 & 2 & $P>10^{-5} / \mathrm{h}$ \\
E & No effect & 2 & 0 & $\mathrm{~N} / \mathrm{A}$ \\
\hline
\end{tabular}

$$
\begin{aligned}
\lambda & =\sigma * \phi, \\
\lambda & =[2.56-5.65] \times 10^{-8} \mathrm{~cm}^{2} / \mathrm{dev} * 2.99 \times 10^{3} \mathrm{n} \cdot \mathrm{cm}^{-2} \cdot \mathrm{h}^{-1} \\
& =[7.65-16.89] \times 10^{-5} / \mathrm{h} .
\end{aligned}
$$

As the reliability of multi-/manycore processors strongly depends on the implemented application (software), the failure rate of the device can be classified within the DO178B/C (Software Considerations in Airborne Systems and Equipment Certification). The $\mathrm{DO}-178 \mathrm{~B} / \mathrm{C}$ is a guideline used as de facto standard for developing avionic software systems [27]. Table 6 shows the level of failure condition.

The failure conditions for avionic systems are described as follows:

(i) Catastrophic: Failure may cause a crash. Error or loss of critical function required to safely fly and land the aircraft.

(ii) Hazardous: Failure has a large negative impact on safety or performance or reduces the ability of the crew to operate the aircraft due to physical distress or a higher workload, or causes serious or fatal injuries among the passengers (safety-significant).

(iii) Major: Failure is significant, but has a lesser impact than a hazardous failure (e.g., passenger discomfort) or significantly increases crew workload (safety related).

(iv) Minor: Failure is noticeable, but has a lesser impact than a major failure (e.g., passenger inconvenience or a routine flight plan change).

(v) No Effect: Failure has no impact on safety, aircraft operation, or crew workload.

Results show that the failure rate of the multicore executing a matrix multiplication as application falls in level D of the DO-178B/C. Therefore, the device is convenient for minor failure conditions, which includes several applications regarding data and image processing. In fact, the NASA's report, "Intelligent Hardware-Enable Sensor and Software 
Safety and Health Management for Autonomous (UAS)" shows the use of Parallella board containing the Epiphany multicore for unmanned aircraft systems [30]. The current article is relevant since it supports the use of the Parallella board in aircraft applications by presenting experimental data concerning its radiation sensitivity.

\section{Conclusions}

Radiation experiments performed with $14 \mathrm{MeV}$ neutrons are a useful technique for evaluating the intrinsic sensitivity of the multicore and dynamic response of the application. Results demonstrate that the Epiphany E16G301 multicore is suitable for embedded systems performing noncritical avionic applications. The fact that the Epiphany does not implement protection mechanisms has permitted a true estimation of the error rate, confirming that protection mechanisms affect testing as stated in [31].

Although the maximum flux provided by the radiation facility is $4.5 \times 10^{7}\left(\mathrm{n} \cdot \mathrm{cm}^{-2} \cdot \mathrm{s}^{-1}\right)$, the applied neutron flux was limited to $7.2 \times 10^{4} \mathrm{n} \cdot \mathrm{cm}^{-2} \cdot \mathrm{s}^{-1}$ in order to avoid perturbations in the circuitry of the Parallella board. However, the applied flux is about eight orders of magnitude greater than the flux at avionic altitudes, which corresponds to almost 10 years of exposure time of the device to neutron radiation at 35,000 feet.

Despite the efforts for protecting the rest of components of the platform, the SD card containing the Linux OS was corrupted in one of the experiments. This was solved by replacing the tainted SD by a new one and repeating the test.

In future work, the Adapteva Epiphany multicore processor will be proposed to be used for image processing in a military aircraft. In parallel, another module containing the Epiphany processor will execute a memory-bound application in order to detect SEUs produced in real operating environment.

\section{Data Availability}

The data used to support the findings of this study are available from the corresponding author upon request.

\section{Disclosure}

The present paper is an extension of the abstract poster presented in the "Nano Science and Nanotechnology Week" held in Quito-Ecuador from 28/08/2017 to 01/09/2017.

\section{Conflicts of Interest}

The authors declare that they have no conflicts of interest.

\section{Acknowledgments}

The authors would like to thank Maud Baylac, Francesca Villa, and Solenne Rey from LPSC for their valuable support during radiation experiments presented in this work. This work has been supported by the Ecuadorian government through the Secretaría de Educación Superior, Ciencia, Tecnología e Innovación (SENESCYT) and the Universidad de las Fuerzas Armadas ESPE (Grant no.PIC-2017-EXT005). Radiation experiments in GENEPI2 at LPSC (CNRS/UGA) have been performed within the "Characterisation Program" of the IRT nanoelec and cofunded by the French government in the frame of the so-called "Programme d'Investissements d'Avenir" under the reference ANR-10-AIRT-05.

\section{References}

[1] R. Hyman, K. Bhattacharya, and N. Ranganathan, "Redundancy mining for soft error detection in multicore processors," IEEE Transactions on Computers, vol. 60, no. 8, pp. 1114-1125, 2011.

[2] E. Kim and N. Shanbhag, "Soft N-modular redundancy," IEEE Transactions on Computers, vol. 61, no. 3, pp. 323-336, 2012.

[3] Certification Authorities Software Team (CAST), "Position paper cast-32 multi-core processors," November 2016, https:// www.faa.gov/aircraft/air_cert/design_approvals/air_software/ cast/cast_papers/media/cast-32A.pdf.

[4] R. Baumann, "Soft errors in advanced computer systems," IEEE Design and Test of Computers, vol. 22, no. 3, pp. 258-266, 2005.

[5] B. Vandana and M. Siva Mukar, "Study of VLSI bulk CMOS and SOI technologies," International Journal of Scientific and Research Publications, vol. 3, no. 2, pp. 1-8, 2013.

[6] G. Gasiot, V. Ferlet-Cavrois, J. Baggio et al., "SEU sensitivity of bulk and SOI technologies to $14 \mathrm{MeV}$ neutrons," IEEE Transactions on Nuclear Science, vol. 49, no. 6, pp. 3032-3037, 2002.

[7] W. Hughes, Single Event Effects Mitigation Techniques Report, DOT/FAA/TC-15/62, Federal Aviation Administration, Washington, DC, USA, 2016.

[8] P. Bieber, F. Boniol, M. Boyer, E. Noulard, and C. Pagetti, New Challenges for Future Avionic Architectures, Onera, Palaiseau, France, AerospaceLab, 2012.

[9] P. Ramos, V. Vargas, R. Velazco, and N. E. Zergainoh, Assessing the Static and Dynamic Sensitivity of a CommercialOff-The-Shelf Multi-Core Processor for Non-Critical Avionic Applications, Nano Science and Nanotechnology Week, Quito, Ecuador, 2017.

[10] S. S. Stolt and E. Normand, "A multicore server SEE cross section model," IEEE Transactions on Nuclear Science, vol. 59, no. 6, pp. 2803-2810, 2012.

[11] S. Guertin, Initial SEE Test of Maestro, National Aeronautics and Space Administration, Jet Propulsion Laboratory, Pasadena, CA, USA, 2012, http://trs-new.jpl.nasa.gov/dspace/bitstream/ 2014/42682/1/12-2145_A1b.pdf.

[12] T. Santini, P. Rech, G. Nazar, L. Carro, and F. R. Wagner, "Reducing embedded software radiation-induced failures through cache memories," in Proceedings of the 19th IEEE European Test Symposium (ETS), pp. 1-6, Paderborn, Germany, May 2014.

[13] D. A. G. Oliveira, P. Rech, H. M. Quinn et al., "Modern GPUs radiation sensitivity evaluation and mitigation through duplication with comparison," IEEE Transactions on Nuclear Science, vol. 61, no. 6, pp. 3115-3122, 2014.

[14] P. Ramos, V. Vargas, M. Baylac et al., "Evaluating the SEE sensitivity of a $45 \mathrm{~nm}$ SOI multicore processor due to $14 \mathrm{MeV}$ neutrons," IEEE Transactions on Nuclear Science, vol. 63, no. 4, pp. 2193-2200, 2016.

[15] V. Vargas, P. Ramos, V. Ray et al., "Radiation experiments on a $28 \mathrm{~nm}$ single-chip many-core processor and SEU error-rate 
prediction," IEEE Transactions on Nuclear Science, vol. 64, no. 1, pp. 483-490, 2017.

[16] H. Quinn, "The use of accelerated radiation testing for avionics," AIP Conference Proceedings, vol. 1525, 2013.

[17] S. Rezgui, R. Velazco, R. Ecoffet, S. Rodriguez, and J. Mingo, "Estimating error rates in processor-based architectures," IEEE Transactions on Nuclear Science, vol. 48, no. 5, pp. 1680-1687, 2001.

[18] F. Miller, C. Weulersse, T. Carriere et al., "Investigation of 14 $\mathrm{MeV}$ neutron capabilities for SEU hardness evaluation," IEEE Transactions on Nuclear Science, vol. 60, no. 4, pp. 2789-2796, 2013.

[19] Jesd89A, Measurement and Reporting of Alpha Particle and terrestrial Cosmic Ray-Induced Soft Errors in Semiconductor Devices, JEDEC Solid State Technology Association, Arlington, VA, USA, December 2016, http://www.jedec.org/sites/ default/files/docs/jesd89a.pdf.

[20] P. Ramos, "Evaluation of the SEE sensitivity and methodology for error rate prediction of applications implemented in multi-core and manycore processors," Ph.D. thesis, TIMA laboratory, Grenoble, France, 2017, http://tima.univ-grenoblealpes.fr/tima/en/mediatheque/PhDthesisresult_id452.html.

[21] F. Villa, M. Baylac, S. Rey et al., "Accelerator-based neutron irradiation of integrated circuits at GENEPI2 (France)," in Proceedings of the Radiation Effects Data Workshop, pp. 1-5, Paris, France, July 2014.

[22] ADAPTEVA, "E16G301 datasheet rev. 14.03.11," December 2016, http://www.adapteva.com/docs/e16g301_datasheet.pdf.

[23] ADAPTEVA, "Epiphany architecture reference rev. 14.03.11," December 2016, http://www.adapteva.com/docs/epiphany_ arch_ref.pdf.

[24] G. Ballard, J. Demmel, O. Holtz, B. Lipshitz, and O. Schwartz, "Communication-optimal parallel algorithm for strassen's matrix multiplication," in Proceedings of the 24th Annual ACM Symposium on Parallelism in Algorithms and Architectures, pp. 193-204, Pittsburgh, PA, USA, June 2012.

[25] ADAPTEVA, "Epiphany SDK Rev. 5.13.09.10," December 2016, http://www.adapteva.com/docs/epiphany_sdk_ref.pdf.

[26] J. Autran, P. Munteanu, P. Roche, and G. Gasiot, "Real-time soft-error rate measurements: a review," Microelectronics Reliability, vol. 54, no. 8, pp. 1455-1476, 2014.

[27] T. Ferrel and D. Ferrel, RTCA DO-178B/EUROCAE ED-12B, Digital Avionics Handbook, McGraw-Hill, New York, NY, USA, 3rd edition, 2014.

[28] V. Hilderman and T. Baghai, Avionics Certification: A Complete Guide to DO-178B (SW), DO-178C (Update), DO254(HW), Avionics Communication Inc., Leesburg, VA, USA, 2013.

[29] W. Youn, S. B. Hong, K. Ryoon Oh, and O. S. Ahn, "Software certification of safety-critical avionic systems: DO-178C and its impacts," IEEE Aerospace and Electronic Systems, vol. 30, no. 4, pp. 4-13, 2015.

[30] K. Rozier, "Intelligent hardware-enable sensor and software safety and health management for autonomous UAS, NASA/ TM-2015-218817," May 2015, https://ntrs.nasa.gov/archive/ nasa/casi.ntrs.nasa.gov/20150021506.pdf.

[31] K. LaBel and L. Cohn, Radiation Testing and Evaluation Issues for Modern Integrated Circuits, NASA/GSFC Code 561.4, 2005. 


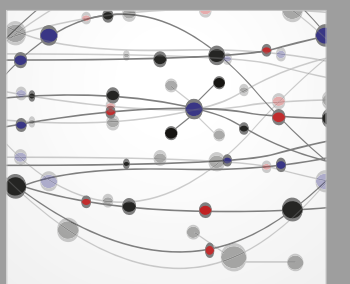

The Scientific World Journal
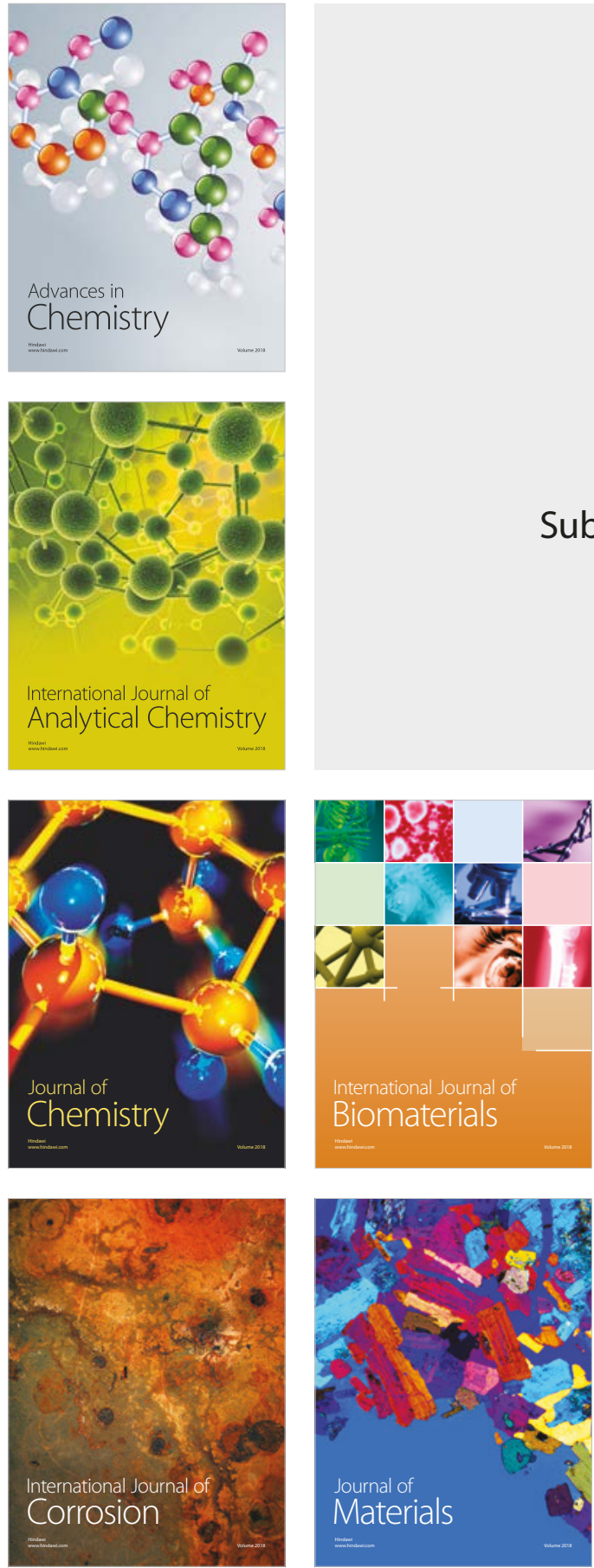

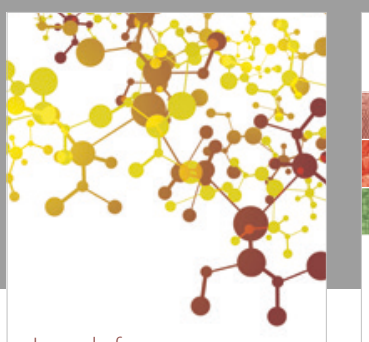

Journal of

Applied Chemistry
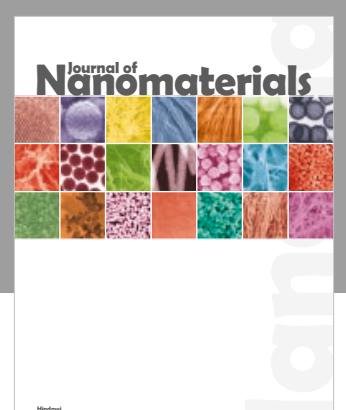

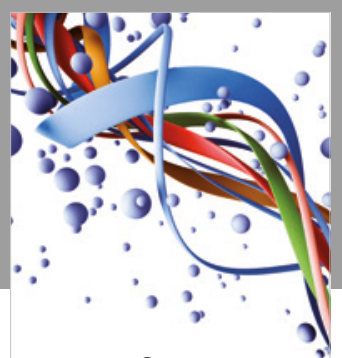

Scientifica

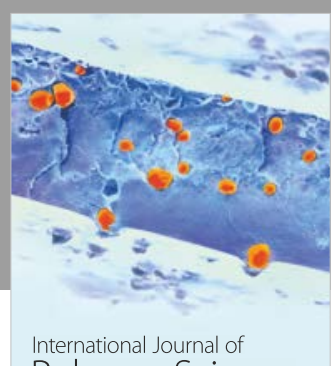

Polymer Science

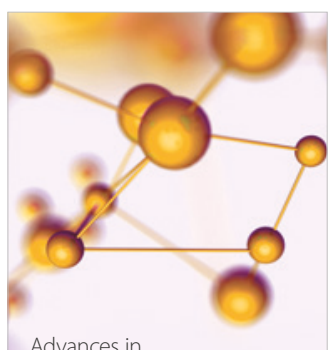

Physical Chemistry
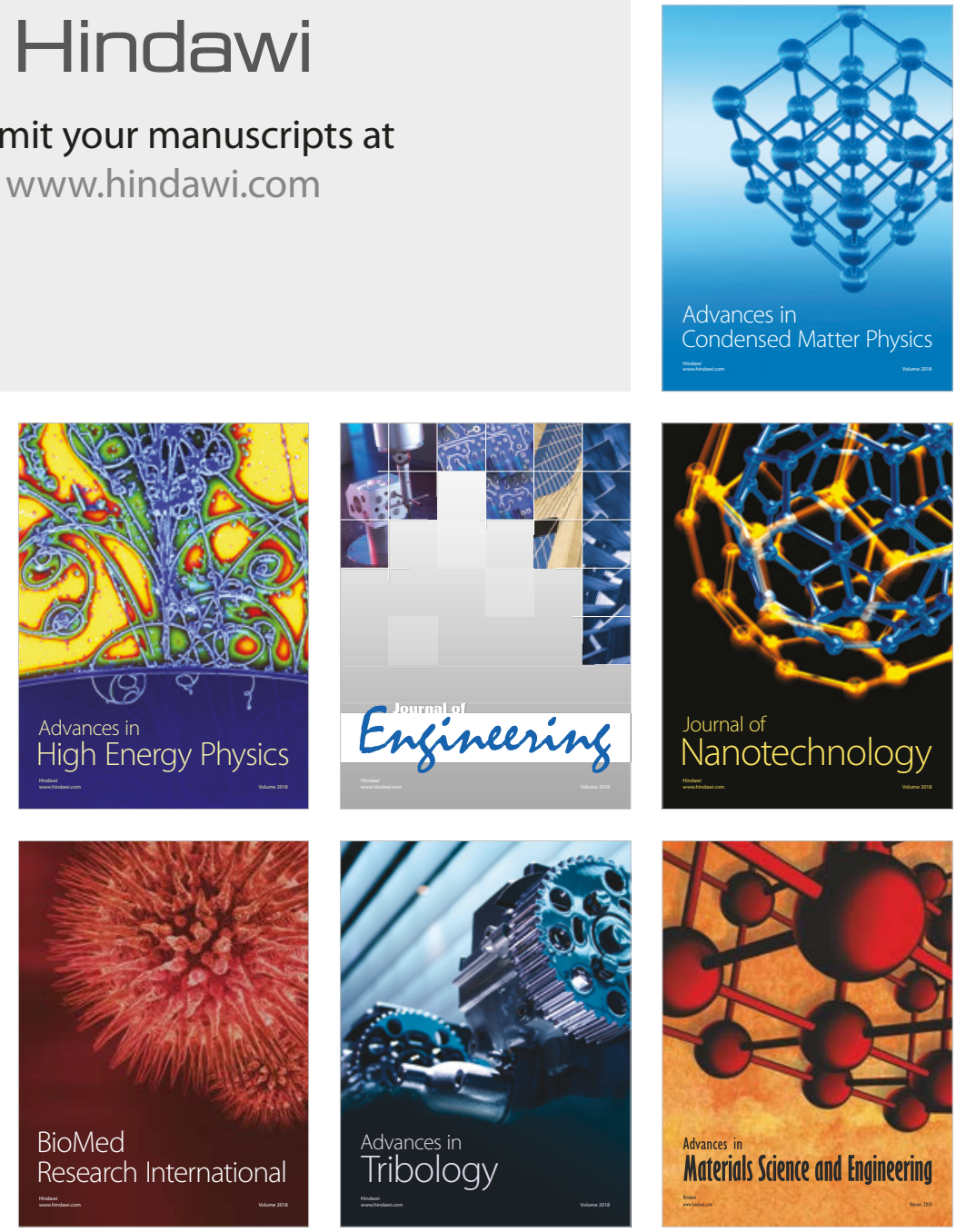